\title{
The ETH Innovation \& Entrepreneurship Lab: Supporting Young Entrepreneurs
}

\author{
Tomas Brenner*
}

\begin{abstract}
For the successful launch and sustainable growth of innovative technologies into business concepts, extensive knowledge and a wide variety of tools are needed. The ETH ieLab provides exactly the right framework and support for this.
\end{abstract}

Keywords: ETH · Innovation · Technology Entrepreneurship

As a melting pot for ambitious tech entrepreneurs, the ETH Innovation \& Entrepreneurship Lab (ETH ieLab), ETH's deep tech incubator, provides tangible support to young entrepreneurs as they transition from scientific innovation and achievement to commercialization and entrepreneurial success. The ETH ieLab is part of the ETH Zurich's technology transfer group (ETH transfer).

Within the framework of the ETH Pioneer Fellowship, which is a grant of CHF 150'000 for 18 months offered to qualified ETH researchers who want pursue an entrepreneurial career, the ETH ieLab offers business advice in the early stages of founding their own companies. Since inception in 2010 , the $100^{\text {th }}$ pioneer fellowship was granted in 2020 .

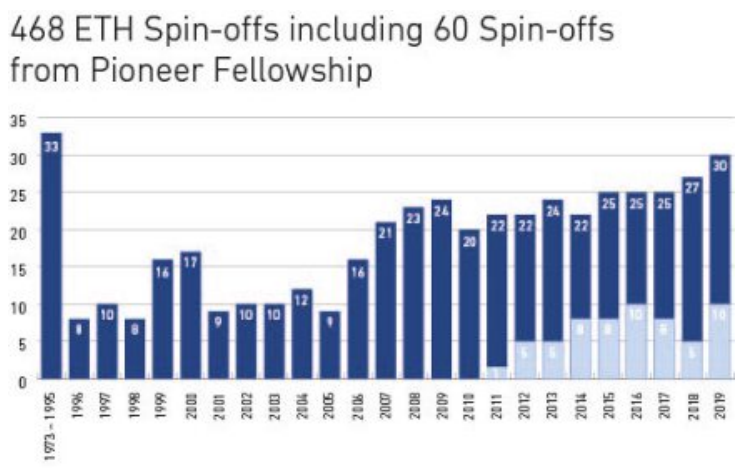

Fig. 1. Successful ETH spin-offs since 1973. Dark blue: 468 ETH spinoffs founded since 1973; light blue: 60 spin-offs from Pioneer Fellowship programme. Source: ETH Zurich, spin-off group. Five-year survival rate: $92 \%$. Detailed statistics: See press release (for electronic version): https://ethz.ch/en/news-and-events/eth-news/news/2020/01/pr-spinoffs-2020.html

Sharing its premises with the ETH Entrepreneur Club, the ETH ieLab offers young entrepreneurs highly practical support. Beyond the free office space and individual coaching sessions, the lab helps them draw up solid business cases - from devising marketing and sales strategies, to defining reliable supply chains, to setting realistic production planning goals.

${ }^{\star}$ Correspondence: Dr. T. Brenner, tomas.brenner@sl.ethz.ch
Through the ETH ieLab, young entrepreneurs benefit from privileged access to experienced coaches, a vast professional partner network for financial, legal, personnel or management issues, and to dedicated, supportive investors.

The numbers speak for themselves: in 2019 alone, ETH entrepreneurs started 30 new companies - not less than 10 of which hail from the Pioneer Fellowship programme. On average, the ETH ieLab is hosting 15-20 Pioneer Fellowship projects. In addition to this, another 5-10 ETH spin-offs are renting office or lab space at the ETH ieLab (Fig. 2).

\section{0 spin-off companies founded at ETH Zurich in 2019 in different fields}

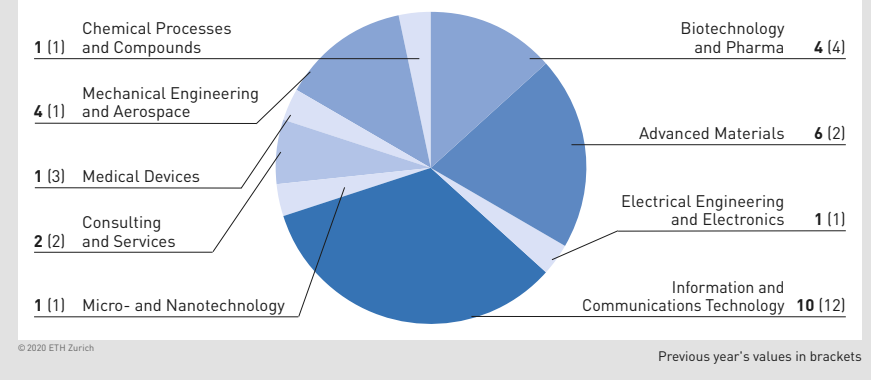

Fig. 2. ETH spin-offs 2019 by field.

The ETH ieLab is the perfect launchpad for recent ETH graduates with entrepreneurial ambitions.

\section{Select examples of Pioneer Fellowship projects and their ETH Spin-offs in the area of chemistry, material science, life sciences}

\section{Oxara}

Cement-free concrete, founded 2019

Oxara developed a cement-free technology that allows the transformation of excavation materials into sustainable concrete.

The construction industry is facing major challenges including scarcity of resources (especially sand), $\mathrm{CO}_{2}$ emissions given extensive cement production, and the increased demand for affordable housing worldwide. Meanwhile, billions of tons of excavation materials end up in landfills every year. This waste material is currently unused and occupies large volumes of space.

Oxara's solution consists of using locally available landfill waste (excavation materials) to produce poured earth concrete. To ensure the process remains simple and competitive to concrete, 
Oxara developed a cement-free concrete technology. This not only enables the casting of the excavation materials in its fresh state using concrete infrastructure, but also accelerates the hardening and subsequent removal of the formwork after 24 hours. Oxara poured earth concrete has all the advantages of concrete, but is 20 times more eco-friendly and 2.5 times less expensive. Once industrialized, it constitutes a sustainable solution for affordable housing and will contribute to the reduction of global $\mathrm{CO}_{2}$ emissions, construction waste, and sand consumption.

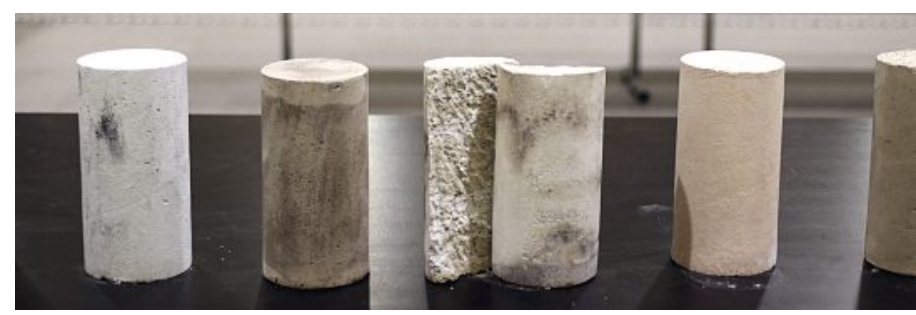

www.oxara.ch

\section{Spectroplast}

\section{$3 D$ silicone print, founded 2018}

With a vision of bringing industrial-scale silicone 3D printing to the mass market, Spectroplast has developed cutting-edge 3D printing technology enabling the use of any off-the-shelf silicone to produce readily-functional products without the use of moulds, thus cutting both costs and time.

Offering the world's first Silicone 3D Printing Service, Spectroplast expands the range of printable materials from rigid to stretchable materials in the world of Additive Manufacturing.

With a very broad field of application, Spectroplast provides customized 3D printed silicone components with highest precision straight out of the printer. Indeed, the 3D printer allows completely new product forms such as patient-specific medical implants, simple sealing rings, tailor-made hearing aids, or artificial heart valves.

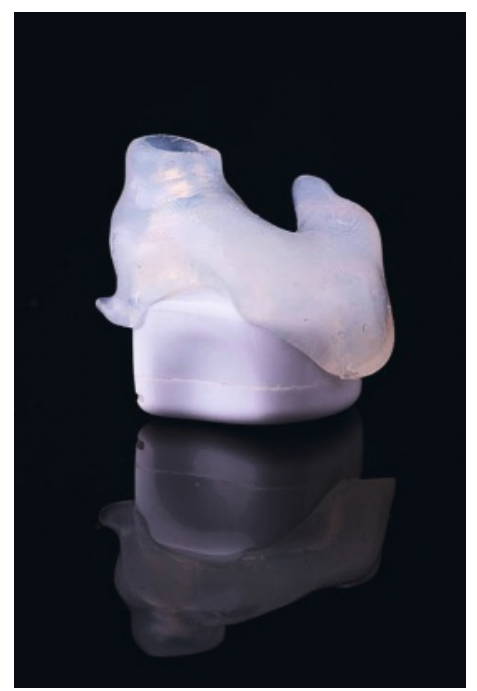

www.spectroplast.com

\section{FenX}

Insulation from ashes, founded 2019

Following the 2016 Paris Agreement on Climate Change, and the 2017 Grenfell Tower fire, there are increasing demands for non-flammable and environmental-friendly insulation materials.

To address this need, and by simply exploiting abandoned and widely-available materials, FenX transforms mineral waste into highly porous foams with excellent thermal properties that com- ply with the newest fire regulations. More importantly, FenX's foams can be fabricated at ambient temperature, thus causing negligible $\mathrm{CO}_{2}$ emissions, and are $100 \%$ recyclable.

With this technology, FenX is addressing the building market (architects, real estate developers, construction companies), offering insulation foams with conventional (panels, bricks) as well as highly specific and totally customized shapes.

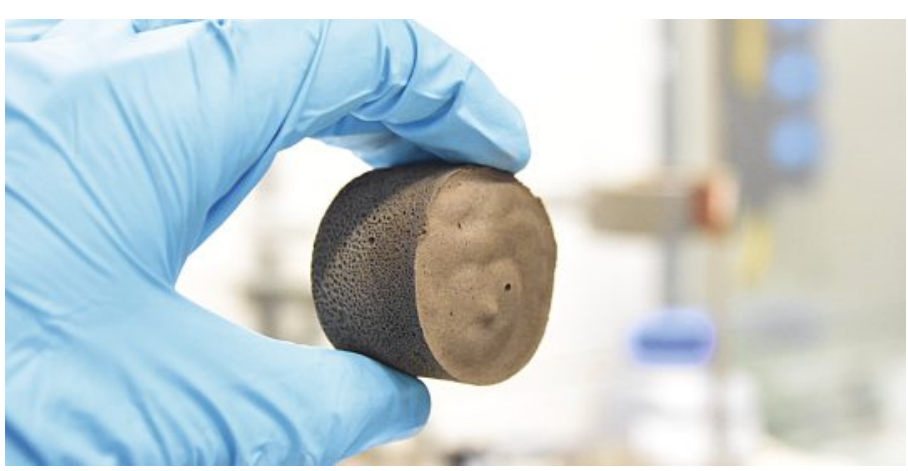

www.fenx.ch

\section{microPow}

Aroma powder, founded 2019

microPow is a micro-foamed, fat-continuous powder with incorporated aroma and flavour compounds. microPow improves the stability and enhances the perception of aroma and flavour compounds in end-products.

The foam-based microstructure stabilizes the contained aromas during processing and storage, enhancing the aroma release (compared to pure aroma compounds). It brings highly stable, natural aromas and structures to a broad product range such as food products (ice-cream, coffee, snacks), cosmetic products (shampoo, washing emulsions, creams), and home-care products (liquid laundry detergent).

microPow is based on an organic, fat-based composition. Free of additives (emulsifiers or stabilizers), its micro-foamed structure allows the incorporation of volatile (gas-soluble), fat-soluble, and suspended aromas, thus generating well-rounded flavour profiles compared to conventional carrier systems for aromas and flavours.

Produced in an energy-efficient process, microPow also actively contributes towards more environmentally-friendly products in food, cosmetics, and home-care products.

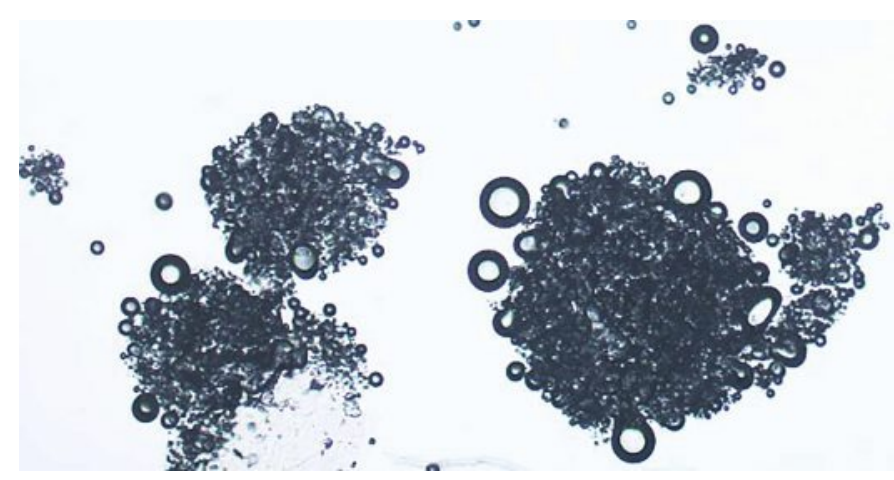

www.micropow.ch

\section{Haelixa}

DNA tracer, founded 2016

Haelixa offers proprietary and innovative solutions to physically mark, trace, and authenticate products from producer to retail, creating transparency along the entire linear or circular supply chain. 
The marker can be applied to raw materials, textiles of all kinds, gold, diamonds, and other products. Haelixa works with retailers, brands, manufacturers, and non-profit organizations.

Along with proving product origins and authentic production, Haelixa protects brands against counterfeiting by providing proof of authenticity and safeguarding the value linked to the brand story, design, processing, and performance.

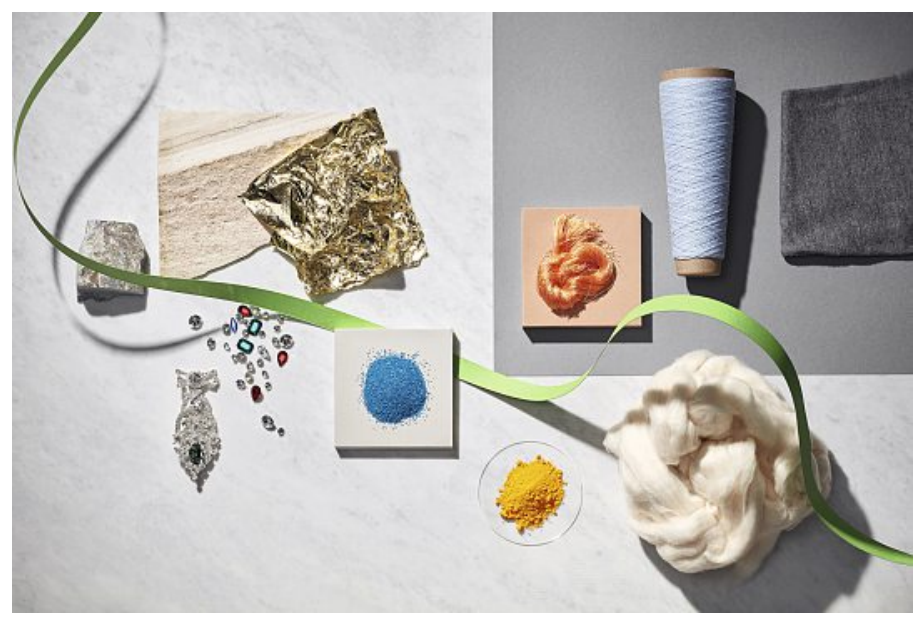

www.haelixa.ch

\section{GUV (Global University Venturing) Award for the ETH transfer Group}

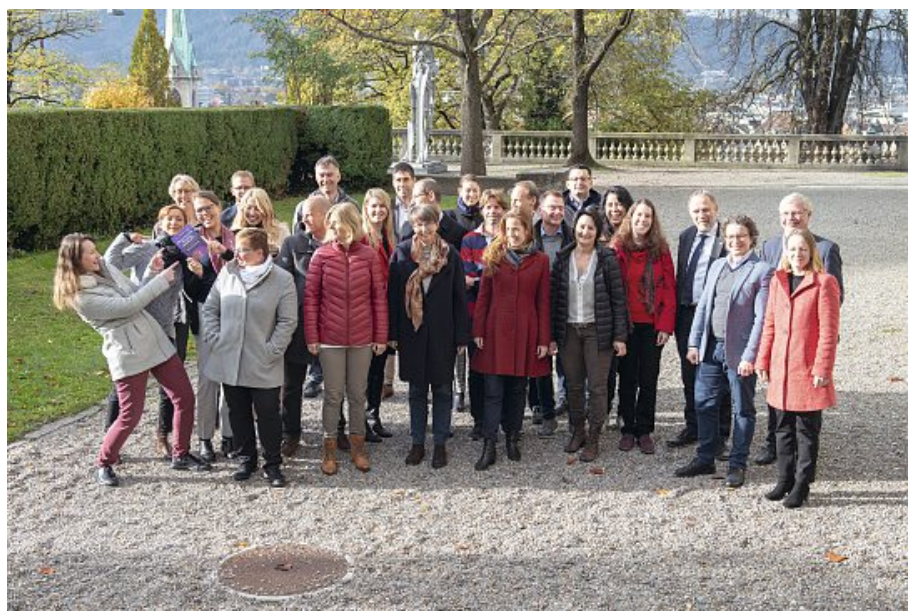

ETH transfer (source: ETH Zurich, Peter Rüegg).

\section{ETH supports Pioneer Fellows and Spin-offs}

ETH ETH transfer is the central Technology Transtransfer fer Office of ETH Zurich of the Vice Presidency Knowledge Transfer and Corporate

Linking science and business Relations. Its activities include: collaboration with industry; the management and commercialisation of ETH's technical intellectual property, and the fostering and support of ETH spin-offs. In 2019, it won the international Global University Venturing (GUV) Award as "Tech Transfer Unit of the Year".

\section{Dr. Silvio} Bonaccio

The ETH ieLab

ETH's deep tech incubator

\section{Dr. Tomas Brenner}

Spin-off group

ETH spinoff support

Dr. Marjan Kraak

\section{ETH}

Foundation

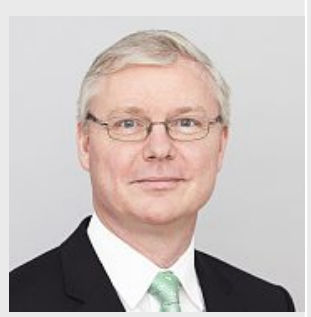

Head ETH Transfer

Rämistrasse 101, CH-8092 Zurich

The ETH ieLab is the home of ETH Pioneer Fellows and ETH spin-offs. The Lab encourages, educates and challenges bright, talented entrepreneurs, supporting them along their path to successfully establishing their own startups.

www.ethz.ch/ieLab

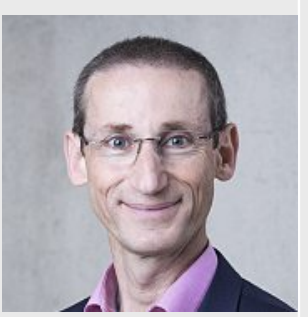

Head of ETH ieLab

Stampfenbachstrasse 52, CH-8092 Zurich

The ETH Zurich spin-off group supports with consulting advice, infrastructure and office/ lab space, as well as contacts during incorporation and for the initial years. It also awards the 'ETH Spin-off' label.

\section{www.spinoff.ethz.ch}

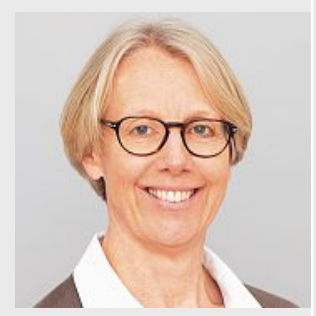

\section{Head ETH}

spin-offs group

Rämistrasse 101 , CH-8092 Zurich

EIH

Foundation

The ETH Foundation supports research and teaching at ETH Zurich. It allocates charitable donations from companies, foundations, and private individuals to ETH Zurich. Every contribution is welcome and helps promote the greatest talents and ground-breaking ideas.

www.ethz-foundation.ch

Received: September 3, 2020

\section{License and Terms}

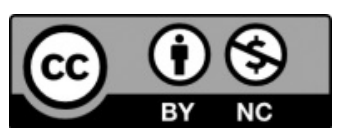

This is an Open Access article under the terms of the Creative Commons Attribution License CC BY_NC 4.0. The material may not be used for commercial purposes.

The license is subject to the CHIMIA terms and conditions: (http:// chimia.ch/component/sppagebuilder/?view=page \&id=12).

The definitive version of this article is the electronic one that can be found at https://doi.org:10.2533/chimia.2020.768 